\title{
Using the MIUU Model for Prediction of Mean Wind Speed At Low Height
}

\author{
Jon Olauson ${ }^{a}, *$, Jonatan Samuelsson ${ }^{b}$, Hans Bergströmc and Mikael Bergkvist ${ }^{a}$ \\ aDivision of Electricity, Department of Engineering Sciences, Uppsala University, Sweden \\ bDepartment of Mathematics, Uppsala University, Sweden \\ 'Department of Earth Sciences, Uppsala University, Sweden
}

\section{Received to be supplied; Revised to be supplied; Accepted to be supplied}

\begin{abstract}
This paper presents an evaluation of using the MIUU mesoscale model to predict mean wind speed at 10 meters above ground. This was done by comparing the model output to 128 meteorological measurements in Sweden. Although some terrain-dependent bias was seen, the model worked surprisingly well. Mean absolute error (MAE) was $0.58 \mathrm{~m} / \mathrm{s}$ or $0.52 \mathrm{~m} / \mathrm{s}$ if a few measurements taken in highly complex terrain were excluded. By using a multiple regression model, taking into account the terrain at site, the latter value was lowered to $0.39 \mathrm{~m} / \mathrm{s}$.

The model described in this paper can be useful for a first evaluation of the wind conditions for small-scale wind power or when measurements are not feasible.
\end{abstract}

Keywords: small-scale wind power, mesoscale model, multiple linear regression, wind resource

\section{INTRODUCTION}

As a part of an interdisciplinary collaboration [1] the feasibility of using small scale wind turbines for generation of emergency power after natural disasters is evaluated. The idea is to use a modified version of the H-rotor vertical axis wind turbine previously developed at the Division of Electricity, Uppsala University [2,3] in a hybrid system with batteries and possibly other energy sources. For this application on-site wind measurements are not an option and it would therefore be advantageous to have tools to assess the wind resource at lower heights. As pointed out in [4] such a tool could also promote development of conventional small-scale wind power, since proper on-site anemometry is very costly.

It has been demonstrated in earlier work [5] that a mesoscale model developed at the former Department of Meteorology, Uppsala University (the MIUU model) show good agreement with wind measurements. The model has however not been thought to correctly capture the wind speeds at low heights since these are more influenced by local topography and vegetation.

The objective of this article is to evaluate the potential of using the MIUU model for a first evaluation of wind conditions at low heights. This is done by comparing the modelled mean wind speed with observed wind speed for 128 measurement stations of the Swedish Meteorological and Hydrological Institute (SMHI). In order to improve the predictions, measurements are classified into categories depending on the surrounding terrain and a statistical model is applied to correct for the discrepancies between MIUU and observations.

The structure of the paper is the following: this introduction section concludes with a description of the MIUU model. In Section 2 data and methods used are described. In Section 3 results are presented and discussed. Finally conclusions are drawn in section 4.

*Corresponding Author: E-mail address: jon.olauson@angstrom.uu.se. 


\section{I.I. The MIUU Model}

The MIUU-model is a three-dimensional mesoscale model with prognostic equations for wind, temperature, humidity and turbulent kinetic energy [6]. Turbulence is parameterised using a level 2.5 scheme following Mellor and Yamada nomenclature. The closure is described in detail in [7]. The MIUU model has a terrain-influenced coordinate system, roughly following the terrain close to the surface and gradually transforming to horizontal at the model top.

At the lower boundary, roughness length and terrain elevation have to be specified at each grid point. The surface temperature is estimated with a surface energy balance routine using as input solar radiation and land use. In the vertical 29 grid points are used. The first one is at the height $z_{0}$, where $z_{0}$ is the roughness length. Close to the ground the vertical grid points are logarithmically separated in order to get high resolution close to the surface, giving 8 computational levels up to $100 \mathrm{~m}$ height, while higher up the grid points become more and more linearly spaced, and the model top is at the height $10000 \mathrm{~m}$, where the vertical resolution is $760 \mathrm{~m}$.

A method to simulate the climatological wind field using the MIUU-model has been developed, the "MIUU-method", reducing the total number of simulations needed [8,9]. With this method a limited number of climatologically relevant simulations are performed, with different wind and temperature conditions. The method is applicable for mapping the wind resources with a resolution of 0.1-10 km. To use this method geostrophic wind (strength and direction), sea and land temperatures, topography, roughness, and land use are needed. No observed boundary-layer winds are needed other than for verification.

A summation over all model runs made (192 runs representing 4 seasons, 3 strengths and 16 directions of the geostrophic wind) and hours (each model run gives an output of 24 hours - a diurnal cycle), gives the climatological mean value for each grid point and each height. Statistics of the horizontal air pressure gradient (the geostrophic wind) are used to weight the results into a longterm climatological average. The statistical background data are based on surface pressure observations from weather stations or on global reanalysis datasets, such as NCEP/NCAR, MERRA or ERA Interim.

\section{DATA AND METHOD}

\section{I. SMHI measurements}

The Swedish Meteorological and Hydrological Institute have been performing wind measurements for a long time at several hundred stations spread across Sweden. The meteorological stations are located to give an undisturbed wind flow, i.e. as little vegetation and other elements as possible in the vicinity. Large parts of Sweden is however covered by forest and consequently many of the met masts have only a small surrounding open area. Prior to 1995 most of the measurement stations were manual but during 1995-1996 over 100 automatic stations were installed. The stations record one 10 minute average value of wind speed and wind direction every three hours. Wind speed resolution is most commonly $1 \mathrm{~m} / \mathrm{s}$, but better in a few cases. The measurements were analysed by SMHI and corrupt data have been removed. When choosing time series to include in the analysis the following principles were applied:

1. Only automatic stations instrumented from 1995 and onwards were included.

2. The series should comprise at least ten whole years of data. The monthly and yearly correlation with reference series (MERRA) is very poor for many stations and therefore long-term correction was considered inappropriate. Accordingly a long measurement period is essential to minimize the influence of long-term variability in mean wind speed.

3. At least $85 \%$ data availability. One series with $78 \%$ availability was however long-term corrected and used. The reason for this was the wish to include an additional coastal station in the analysis.

4. Only stations with measurement height 10 meters above ground. A few measurements are carried out in lighthouses at other heights but these have been rejected, partly because the height is sometime unknown and partly because the wind flows are expected to be disturbed by the buildings. The National Road Administration (Vägverket) are also performing measurements at 6 meters above ground. These have been rejected because of the low height and the disturbance from vehicles. Using only one measurement height also facilitates the statistical analysis of the results. 
In total 128 stations were included in the analysis. The data series were truncated to whole years. 119 of the resulting measurement series spans 15-17 years and 9 spans 11-14 years. The data availability is generally high; average value is $98 \%$.

When training and evaluating the statistical model, the mean wind speeds for the SMHI measurements were considered to be true long-term means. For various reasons this is obviously not entirely correct. The uncertainty and possible bias in the measurements will lead to increased errors in the evaluation and possibly also a biased model. The most important sources of uncertainties are listed below.

1. Measurement errors including icing. The measurements considered in this work were performed with cup anemometers (heated if appropriate) and ultrasonic anemometers. Service of the anemometers was performed annually if no unexpected errors occurred. As mentioned above, the recorded data has been reviewed by SMHI. According to SMHI, the most severe icing problems can be expected for measurements in "bare mountain" terrain (see Section 2.3), which, for other reasons, were excluded from the statistical modelling.

2. Sampling frequency. For most of the measurements, ten minute average samples were recorded every three hour. The three hour sampling frequency reduces the amount of available data to around 30-50 000 samples for each station and no information was available for certain hours. This is however not believed to be a major issue since the diurnal patterns (if any) were smooth.

3. Local obstacles. According to the WMO guidelines [10], measurements should be representative for the area and not be disturbed by local obstacles such as trees or buildings. Although SMHI strives to meet these demands, it is not always possible in the densely forested Sweden. The effect of obstacles was implicitly accounted for in the statistical model by classifying the measurements according to their surrounding vegetation.

4. Long-term variability. Although all measurements span at least eleven years (and most more than 15 years), there might exist patterns on an even longer time scale so that the recorded mean wind speed deviates from the true mean. The growth and potential clearcutting of forests can also induce long-term trends.

Based on the comments to the abovementioned sources of uncertainty, it seems reasonable to a assume that the measurements included in the statistical model are not seriously biased. The measurement uncertainty will however contribute to the errors in the evaluation of the model (so in fact, the model is somewhat better than the evaluation tells us). The deterioration of model performance from adding additional noise to the data is quantified in Section 3.1.

\subsection{Wind speed interpolation}

For the surface layer (up to around 50-150 meter above ground) an equation for the vertical wind profile can be derived using physical considerations, the "logarithmical law". An alternative used in this work is the empirically derived "power law"

$$
u(z)=u\left(z_{r e f}\right)\left(\frac{z}{z_{r e f}}\right)^{\alpha},
$$

where $u$ is the wind speed, $z$ is the height above ground and $\alpha$ is the shear exponent, which is a function of surface roughness, orography, atmospheric stability etc. [11]. Inside or near forests, $z$ and $z_{\text {ref }}$ should be reduced by the parameter $d$, the displacement height. The displacement height inside the forest can be parameterised by canopy height, tree breadth and distance between trees [12], but often simply $2 / 3-1$ of forest height is used as a value for $d$. Outside the forest, the displacement height will gradually decay towards zero.

Near ground, the wind speed is heavily affected by nearby obstacles and the wind profile is notoriously hard to predict, even if it would be feasible to make visits to all 100+ sites. The approach used in this work was to assume zero displacement height, make a categorisation of the measurement based on the surrounding terrain (see section 2.3) and then apply a statistical model to adjust the mean wind speed. In other words no attempt was made to make an analysis of the displacement height at each site. 
The MIUU-model results used were from the database developed making the Swedish wind resource mapping [5], with horizontal resolution of $1 \mathrm{~km}$. For vertical extrapolation MIUU wind at 12 and 21 meters were used with Eq. 1 in order to calculate the wind at $10 \mathrm{~m}$. The wind shear in MIUU relatively closely follows the power law, so the error in the extrapolation from 12 to $10 \mathrm{~m}$ is expected to be very small. For horizontal interpolation the bilinear method was used.

\subsection{Terrain type classification}

As mentioned above no attempt was made to estimate the actual displacement heights at the measurement locations. Instead the data was divided into five categories depending on the terrain type around the measurement stations: coastal, open landscape, forest, urban and bare mountains. When sorting the stations into categories the dominating terrain type within a radius of 700 meters was estimated from satellite photos. This radius was (somewhat arbitrarily) chosen based on the resolution of the MIUU model and the scale relevant for the displacement height decay outside a forest. Since measurements in urban terrain and forest have a lot in common, these measurements were grouped together into the class "canopy" in the statistical modelling. Only nine observations in bare mountain terrain were available which was considered too few for a robust statistical modelling. Therefore these stations were excluded from the statistical analysis. Information on the terrain type classification is given in Table 1.

\subsection{Long-term correction}

Since the wind climate varies from year to year it might be necessary to long-term correct (LTC) shorter wind measurements to get a representative mean wind speed. For the SMHI measurements analysed the average standard deviation in yearly mean wind speed is around $8 \%$. Several different LTC methods and reference series are available. In this paper the linear regression method was used (see [13] for a method description) with the MERRA reanalysis dataset [14] as long-term reference.

Generally the SMHI measurements show a low, in a few cases even negative, correlation to MERRA in monthly and yearly mean wind speed. Because of this and since at least 10 years of data were available for the selected stations, it was decided to not long-term correct the measurements. One exception was made for a coastal station with a bit low data availablility (78\%) and good correlation to MERRA. The adjustment in mean wind speed for this station was however very small.

The main use of LTC in this paper was for a comparison of the model results to the uncertainties resulting from a measurement of length 1-12 months (see Section 3.3). For this purpose it turned out that better results were achieved by using only one directional bin instead of the 12 or 16 normally used.

Table I. Information on the terrain type classification of measurement masts

\begin{tabular}{llc}
\hline Terrain & Comment & Count \\
\hline Coastal & $\begin{array}{l}\text { All stations are relatively exposed to open sea. Stations near lakes } \\
\text { were not classified as coastal (rather open landscape if water was } \\
\text { the dominating feature). }\end{array}$ & 20 \\
Open landscape & $\begin{array}{l}\text { Could be located in agricultural areas or on airports and generally } \\
\text { have elements of forest within the 700 m radius. }\end{array}$ & 31 \\
Forest & Often located in a smaller open areas in a forest landscape. & 56 \\
Urban & $\begin{array}{l}\text { Generally located in an open area in the outer parts of a city, } \\
\text { with relatively low buildings in the vicinity. }\end{array}$ & 12 \\
Bare mountain & Bare mountains are defined as mountains above the vegetation \\
& $\begin{array}{l}\text { line, around 600 to 900 m above sea level for northern to middle } \\
\text { Sweden. These sites are also characterized by complex orography }\end{array}$ & 9 \\
& which may not be resolved in mesoscale models. & \\
\end{tabular}




\subsection{Statistical model}

2.5.I. Basic model

The basic idea behind the statistical model is to linearly adjust the MIUU model so that it better fits the SMHI data. In order to do this the data was grouped by terrain type and modelled by

$$
f_{t k}\left(u_{k}\right):=b_{1, t k} u_{k}+b_{0, t k} \text {, }
$$

where $u_{k}$ denotes the (MIUU) modelled wind speed and $t_{k} \in\{1,2,3\}$ the terrain type, where 1 corresponds to coastal, 2 to open landscape and 3 to canopy, at observation point $k \in\{1, \ldots, n\}$.

\subsubsection{Improved model}

While the model in Section 2.5.1 is fine for most of the observations, it relies heavily on that all observations are and can be correctly classified as belonging solely to one of the three used terrain types. This was the case for most $(86 \%)$, but not all of the observations in the data set. Our method to deal with the trickier observations was to assign them one major and one minor terrain type and let the improved model for these observation be a linear combination of the basic models for the each of the two terrain types. We balanced this linear combination using the weights $2 / 3$ for the major terrain type and $1 / 3$ for the minor.

Example. Station 6204 was considered to be mostly canopy but also partly open (major terrain canopy, and minor terrain open, that is). Therefore, the improved model for station 6204 would be:

$$
f(\cdot)=\frac{2}{3} f_{3}(\cdot)+\frac{1}{3} f_{2}(\cdot)
$$

It turns out (see Section 2.5.3) the improved model can be interpreted as a simple case of multiple regression, i.e:

$$
\mathrm{f}(\mathrm{U})=\mathrm{U} \beta
$$

using the $n$-by- 6 design matrix

$$
\mathrm{U}:=\left(\begin{array}{cc}
u_{1} \alpha_{1}^{T} & \alpha_{1}^{T} \\
\vdots & \vdots \\
u_{n} \alpha_{n}^{T} & \alpha_{n}^{T}
\end{array}\right)
$$

where $\alpha_{k}$ is the vector

$$
\alpha_{k}:=\left(\begin{array}{lll}
\alpha_{k, 1} & \alpha_{k, 2} & \alpha_{k, 3}
\end{array}\right)^{T}
$$

for

$$
\alpha_{k, t}:= \begin{cases}1 & ; t \text { is the sole terrain of obervation } \mathrm{k} \\ 2 / 3 & ; t \text { is the major terrain of obervation } \mathrm{k} \\ 1 / 3 & ; t \text { is the minor terrain of obervation } \mathrm{k} \\ 0 & ; \text { otherwise }\end{cases}
$$

and $\beta$ is the $6-$ by- 1 vector to be fitted. The software $\mathrm{R}$ was used for the statistical modelling (the code used could be sent upon request).

2.5.3. Multiple regression property derivation

Using the definitions made in Section 2.5.1, but with $m$ different terrain types, define

$$
\alpha_{k}=\left(\alpha_{k, 1} \cdots \alpha_{k, m}\right)^{T}
$$


as the vector of weights, indicating how appropriate each terrain type is for describing the surroundings of station $k$. Furthermore, assume $\alpha_{k}$ to have the following properties (this can be done without loss of generality):

$$
\begin{gathered}
\sum_{i=1}^{m} \alpha_{k, i}=1 \text { for all } k \in\{1, \ldots, n\} \\
0 \leq \alpha_{k, i} \leq 1 \text { for all } k \in\{1, \ldots, n\}, i \in\{1, \ldots, m\}
\end{gathered}
$$

We can now, in accordance with what is stated in the first part of Section 2.5.2, define the improved model as

$$
\begin{aligned}
f\left(u_{k}\right): & =\sum_{i=1}^{m} \alpha_{k, i} f_{i}\left(u_{k}\right)=\sum_{i=1}^{m} \alpha_{k, i}\left(b_{1, i} u_{k}+b_{0, i}\right)=\sum_{i=1}^{m} \alpha_{k, i} u_{k} b_{1, i}+\sum_{i=1}^{m} \alpha_{k, i} b_{0, i} \\
& =\left(\begin{array}{lllllllll}
\alpha_{k, 1} u_{k} & \cdots & \alpha_{k, m} u_{k} & \alpha_{k, 1} & \cdots & \alpha_{k, m}
\end{array}\right)\left(b_{1,1} \cdots b_{1, m} b_{0,1} \cdots b_{0, m}\right)^{\mathrm{T}},
\end{aligned}
$$

which, for the whole data set, can be written in the matrix form

$$
\mathrm{f}(\mathrm{U})=\mathrm{U} \beta,
$$

where $\mathrm{u}$ denotes the vector of (MIUU) modelled data, $\beta$ is a $2 m$-by- 1 vector to be fitted, and $\mathrm{U}$ is the design matrix given by Eq. 5. This shows that the improved model is, in fact, just a simple case of multiple regression. The result is general and could be applied to any weigths satisfying equations (9) and (10) and any number of terrain types.

\section{RESULTS AND DISCUSSION}

\section{I. MIUU raw data}

Figure 1 shows the mean wind speed from the MIUU model at 12 and 33 meters above displacement height and observed wind speed $10 \mathrm{~m}$ above ground. Model and measurements correspond well in the general trend that high mean wind speeds can be found in the mountain ridge (middle to northern Sweden, westernmost part), along the coastline and, to some extent, close to the major lakes. In the inland the mean wind speed is however low.

In Figure 2 scatter plots with MIUU and observed mean wind speed for the different terrain types are shown. The filled circles represent sole terrain and open circles major terrain as defined in Section 2.5.2. It could be noted that observed wind speed are lower than modelled in forest and urban environment. This is expected since a zero-plane displacement is present in these areas. In open landscape there is no obvious systematic difference between model and observations. It is however clear that observation with open landscape as the sole terrain type lies above those which has been classified as partly forest or urban. This result strengthen the idea to use multiple regression instead of separate regression models for the different terrain types.

Wind speeds in the most complex terrain ("bare mountains") are unsurprisingly the most difficult to model. The difference between actual elevation and elevation as seen by the model was up to almost 200 meters at the studied sites. In average the elevation of the stations were just over 30 meters above the model elevation. More surprising is perhaps the systematic difference between model and observations in coastal areas. A possible explanation is that most of the coastal met masts are deployed on small islands in open water, often in the order of 10 meters elevation. These islands are not captured by a model with horizontal resolution $1 \mathrm{~km}$.

\subsection{Statistical model}

The statistical modelling was based on 68 stations with "canopy" as their sole or main terrain, 20 coastal stations and 31 stations in open landscape. One canopy station was removed from the analysis, considered an outlier based on the high leverage (extreme value of the independent variable such that the lack of neighboring observations means that the fitted regression model will 


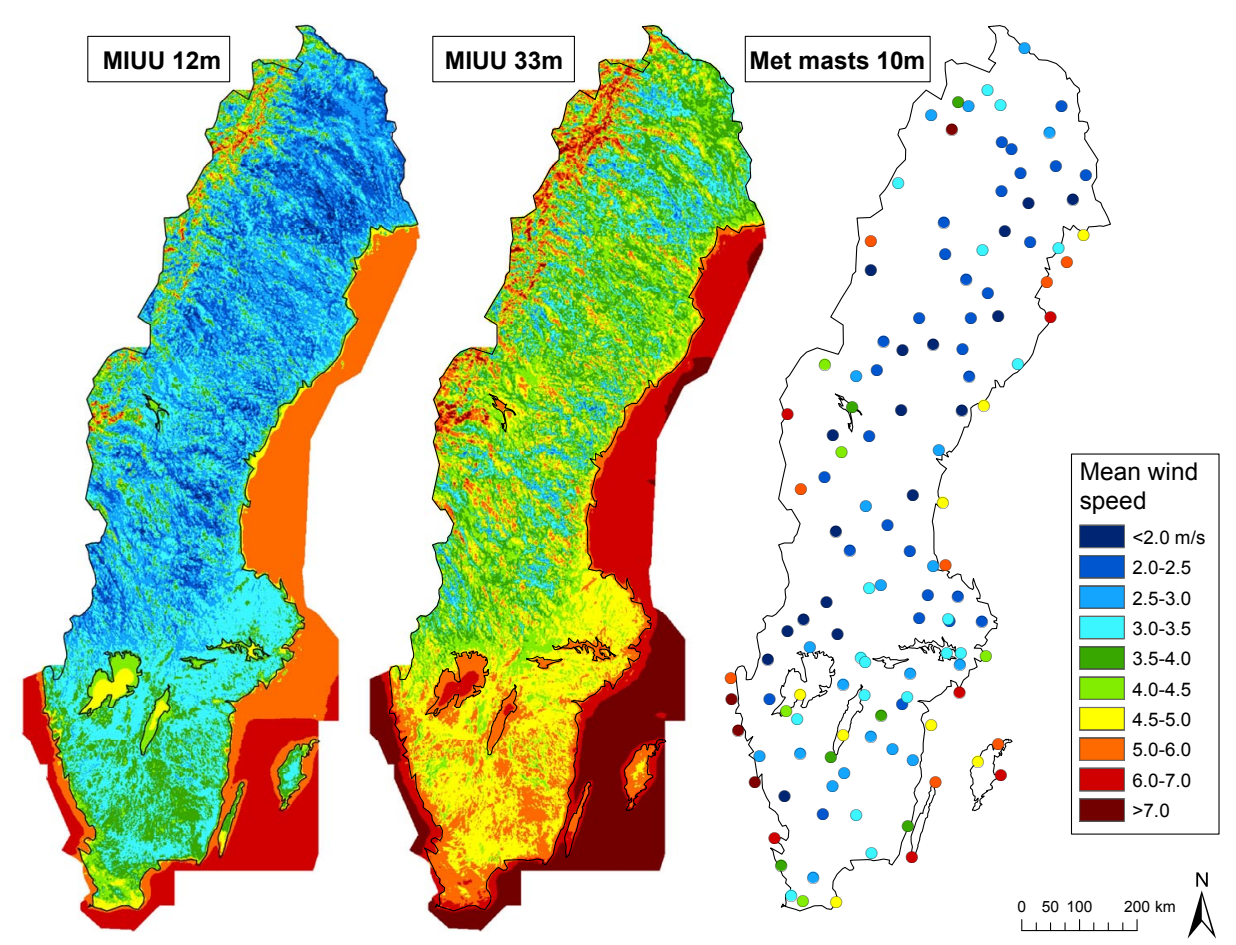

Figure I. Mean wind speed from the MIUU model at 12 and 33 meters above displacement height and observed wind speed $10 \mathrm{~m}$ above ground
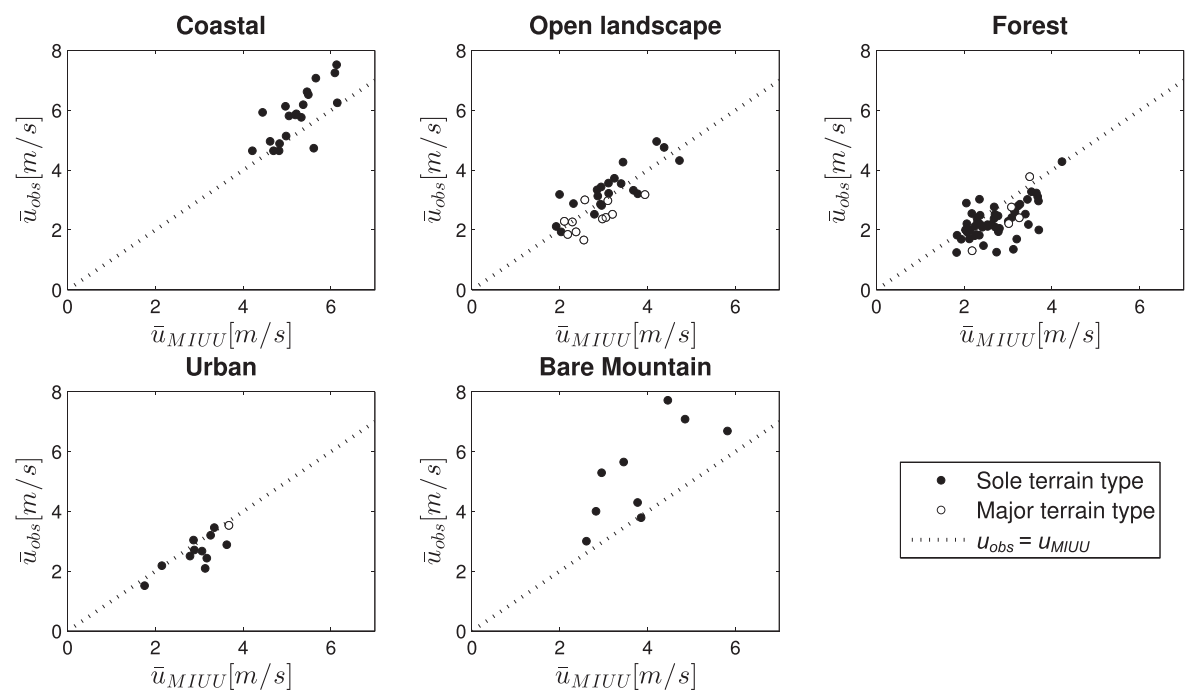

Figure 2. Scatter plots with MIUU wind speed on the horizontal axis (10 $\mathrm{m}$ above displacement height) and observed wind speed on the vertical axis ( $10 \mathrm{~m}$ above ground). The data is grouped according to terrain classification of the met mast

pass close to that particular observation). The removed observation was the forest station with highest mean wind speed in Figure 2. Apart from this observation the material was found robust; near normal distribution of residuals and no severe trends in residuals as a function of fitted mean wind speed.

The errors were calculated with the leave-one-out cross-validation (LOOCV) method. This means that the error for each observation was calculated with a model trained on all data except the observation. In order to evaluate the robustness of the analysis, noise was added to the MIUU mean wind speed and new models were trained. The standard deviation of the added noise was varied 


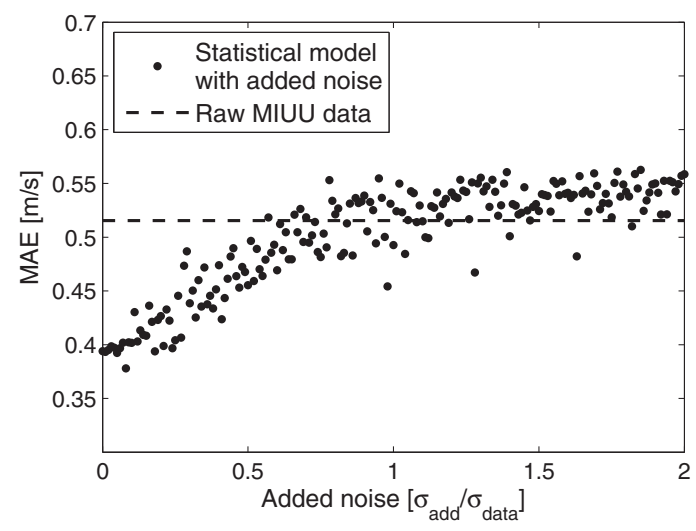

Figure 3. Sensitivity analysis. Noise was added to the MIUU data and the resulting mean absolute error calculated

from 0 up to 2 standard deviations of the underlying data. Figure 3 show the results of the analysis. The errors are in the same order as for raw MIUU data with around 0.8 added standard deviations, which proves the robustness of the statistical model.

Table 2 show different types of error metrics for raw MIUU data and after the statistical correction was performed. Even without the statistical correction MIUU performs relatively well with mean absolute error (MAE) of $0.52 \mathrm{~m} / \mathrm{s}$, or $0.58 \mathrm{~m} / \mathrm{s}$ if the measurements in bare mountain terrain are included. The statistical model reduces the error with $24 \%$ to $0.39 \mathrm{~m} / \mathrm{s}$. For comparison the last column contain results for the statistical model when LOOCV is not used, i.e. the model is trained and evaluated using the same data. A relevant comparison is also [4] who evaluated a semiempirical model to predict mean wind speed and energy yield for 38 sites in UK. By optimising some parameters they achieved a MAE of $0.52 \mathrm{~m} / \mathrm{s}$ or $16 \%$.

The optimised expression for mean wind speed $10 \mathrm{~m}$ above ground as a function of MIUU wind speed and terrain type was

$$
\bar{u}=\left(1.2150_{\alpha 1}+0.9201_{\alpha 2}+0.5656_{\alpha 3}\right) \cdot \bar{u}_{M I U U}-0.5142_{\alpha 1}+0.3564_{\alpha 2}+0.7573_{\alpha 3},
$$

where $a$ with subscript 1-3 are terrain coefficients for coastal terrain, open landscape and canopy respectively, taking one of the values $0,1 / 3,2 / 3$ or 1 as described in Section 2.5.2. Using this expression yields the scatter plot shown in Figure 4. The largest errors in $\mathrm{m} / \mathrm{s}$ are seen for the costal stations, followed by open landscape and canopy. For the relative errors (in $\%$ of observed mean wind speed) the order is the opposite.

\subsection{Comparison with short wind measurements}

The proposed method is intended as an alternative or a supplement to on-site wind measurements. It is therefore relevant to compare the accuracy of the method with the uncertainty related to relatively short measurements. For commercial scale wind power projects measurement campaigns are conducted for at least one whole year, but often longer. For small-scale wind power the measurements are generally taken during a shorter time period (and with cheaper/less accurate equipment).

Table 2. Model errors for MIUU and for combined model (i.e. MIUU with statistical correction). Subscript "all" indicates all 128 measurements and subscript " $s$ " the I 18 measurements included in the statistical modelling. Subscript LOOCV stands for “leave-one-out cross-validation”. Positive bias implies a model overestimation. Percentage MAE errors are given relative the observed value

\begin{tabular}{lcccc}
\hline & MIUU $_{\text {all }}$ & MIUU $_{\mathrm{s}}$ & Combined $_{\mathbf{s}}$, LOOCV & Combined $_{\mathbf{s}}$ \\
\hline MAE & $0.58 \mathrm{~m} / \mathrm{s}$ & $0.52 \mathrm{~m} / \mathrm{s}$ & $0.39 \mathrm{~m} / \mathrm{s}$ & $0.37 \mathrm{~m} / \mathrm{s}$ \\
MAE $(\%)$ & $20 \%$ & $20 \%$ & $15 \%$ & $14 \%$ \\
RMSE & $0.78 \mathrm{~m} / \mathrm{s}$ & $0.65 \mathrm{~m} / \mathrm{s}$ & $0.51 \mathrm{~m} / \mathrm{s}$ & $0.47 \mathrm{~m} / \mathrm{s}$ \\
Bias & $+0.01 \mathrm{~m} / \mathrm{s}$ & $+0.12 \mathrm{~m} / \mathrm{s}$ & $-0.00 \mathrm{~m} / \mathrm{s}$ & $-0.00 \mathrm{~m} / \mathrm{s}$ \\
\hline
\end{tabular}




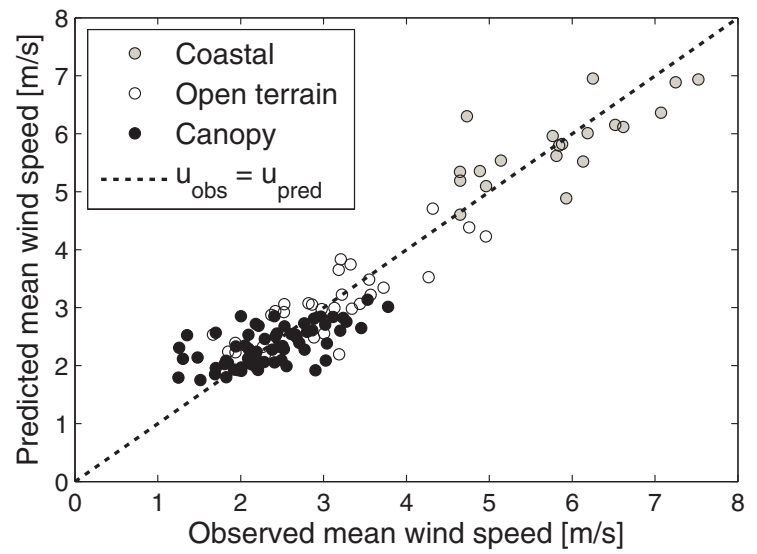

Figure 4. Scatter plot with predicted and observed wind speed. Observations are grouped according to their main terrain classification

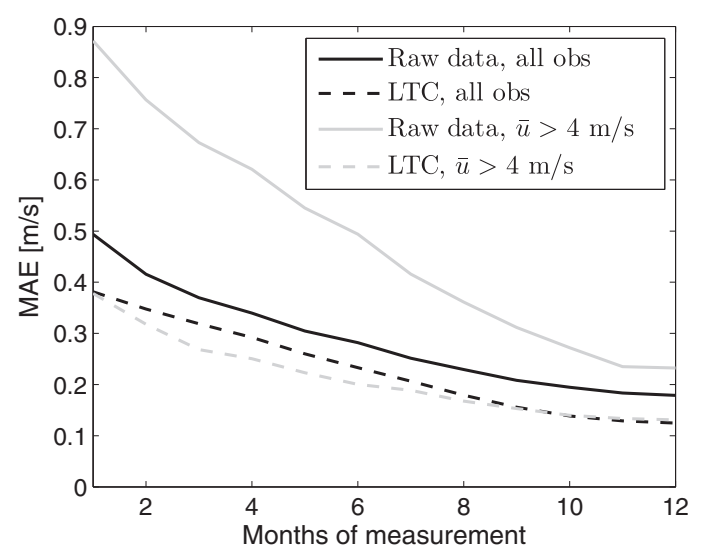

Figure 5. Mean absolute error using a short measurement period (I-12 months). Errors are given for "raw" data and long-term corrected data. Errors for stations where mean wind is above $4 \mathrm{~m} / \mathrm{s}$ are plotted separately

Figure 5 show the errors related to 1-12 months of measurement. One hundred random samples were taken for each measurement length (integer months) and station, and the mean wind was compared to that of the whole 11+ years measurement period. Results are given for all observations included in the statistical model (118 stations) as well as for the stations with observed wind speed above $4 \mathrm{~m} / \mathrm{s}$. The results are given both for the use of raw data and long-term corrected data, since it is not certain that a proper LTC will be performed for small-scale projects.

As already mentioned the correlation between wind measurements and MERRA reference data is generally poor. The benefit of using LTC is therefore relatively small. For stations with higher mean wind speed the correlation is however much better and the LTC is consequently more successful in reducing the errors.

The MAE of the combined model is $0.39 \mathrm{~m} / \mathrm{s}$ for all observations and $0.55 \mathrm{~m} / \mathrm{s}$ for observations with mean wind speed above $4 \mathrm{~m} / \mathrm{s}$. This is equivalent to around 2.5 months and 5 months respectively of measurement without LTC. If long-term correction is used the corresponding period needed to "beat" the model is 1 month and less than one month respectively.

\subsection{Wind energy}

So far only mean wind speed has been discussed. Of course this information alone is not sufficient to predict the energy yield for a particular site; the distribution of wind speeds are also of importance. The two-parameter Weibull distribution is commonly used:

$$
p(u)=\frac{k}{A}\left(\frac{u}{A}\right)^{k-1} \cdot \exp \left[-\left(\frac{u}{A}\right)^{k}\right]
$$




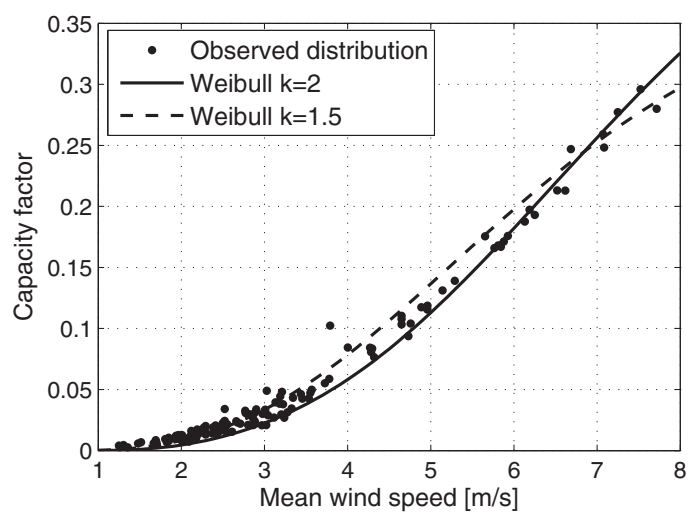

Figure 6. Capacity factor with observed distribution of wind speeds and Weibull distributions based on observed mean wind speed. Power curve for the Bergey $10 \mathrm{~kW}$ wind energy converter was used in the calculation and 10\% losses were included. The capacity factor is given relative the maximum power $(12.5 \mathrm{~kW})$

where $p$ is the probability density function of wind speed $u$, given the Weibull scale factor $A$ and shape factor $k$. For a given mean wind speed and shape factor, the Weibull scale factor can be computed. In the MIUU model Weibull parameters are calculated for each grid point. These are however not publicly available and were therefore not included in the analysis. The question is thus how large uncertainty this lack of information induces on the wind energy calculations?

As also noted in [15] there is a positive correlation between mean wind speed and Weibull shape parameter. The Rayleigh distribution, i.e. a Weibull distribution with shape factor 2, is often a good approximation for sites with higher mean wind speed, but for sites with mean wind speed below $3.5 \mathrm{~m} / \mathrm{s}$ a shape factor of around 1.5 or lower seem more appropriate. This is highlighted in Figure 6 where the capacity factor (mean power divided by rated power) calculated with observed wind speed distribution is compared to capacity factor with Weibull distributed wind speed. For sites with low mean wind speed the relative error using Weibull distributed wind can be large. The absolute deviations from capacity factors calculated with Weibull distributed wind are however relatively small for all measurements except one.

\section{CONCLUSIONS}

The MIUU mesoscale meteorological model and the MIUU method can be used to generate wind climatologies. Contrary to what has previously been thought the model proved to give relatively accurate predictions of the mean wind speed at 10 meters above ground, as compared to data from 128 meteorological stations in Sweden. Measurements in or near forest and urban terrain generally show low mean wind speed. Without taking into account the displacement height the MIUU model, as expected, overpredicts these winds. In open landscape there is no obvious bias in MIUU predictions, but coastal stations generally have higher mean wind speed than anticipated from MIUU. For measurements in highly complex "bare mountain" terrain, the errors were much larger than for other terrain types. The mean absolute error in the predictions were $0.58 \mathrm{~m} / \mathrm{s}$ or $0.52 \mathrm{~m} / \mathrm{s}$ if the measurements taken in bare mountain terrain were excluded.

By correcting the predictions with a multiple regression model the results were improved further; the mean absolute error was reduced with $24 \%$ to $0.39 \mathrm{~m} / \mathrm{s}$. It was found that in order to get the same accuracy, several months of on-site measurements can be required. The model is simple to use, only requiring information on terrain type at the site, and should therefore be suitable even for persons without deeper knowledge in wind engineering. The combined model can be used to complement on-site measurements or, if measurements are not viable, replace those.

It was also found that energy calculations based on Weibull distributed wind speeds corresponded well to calculations based on observed distributions. When the mean wind speed is low a Weibull shape factor of 1.5 or lower is proper, while the Rayleigh distribution could be used when the mean wind speed is above around $5 \mathrm{~m} / \mathrm{s}$.

One must though be cautious when using the model. Firstly the model is not applicable in very complex terrain. Secondly one must bear in mind that meteorological measurements are performed in as open terrain as possible (although for Swedish conditions this could mean quite a lot of forest 
in the vicinity). The same principle should be applied when using the model to find suitable sites for small-scale wind turbines. Finally the model is only valid for mean wind speeds 10 meters above ground. Although one can argue that it is likely that similar trends will be seen at other low heights, no such claims could be made based on this paper.

\section{ACKNOWLEDGEMENTS}

The authors would like to thank Marcus Flarup and Sverker Hellström at SMHI for provision of data and kind help with information on the measurement stations. This work was conducted within the StandUP for Energy strategic research framework. The study was funded by the Swedish Civil Contingencies Agency (MSB) through contract 2010-2787. The Swedish wind resource mapping using the MIUU-model was financed by the Swedish Energy Authority.

\section{REFERENCES}

[1] S. Halldin, L. - $\AA$. Larzon, D. Nohrstedt, L. Nyberg. and S. Ullberg, Science plan for the centre for natural disaster science (CNDS), Tech. Rep. Version 1.0 (Jul. 2011).

[2] A. Goude, Fluid mechanics of vertical axis turbines: Simulations and model development, dissertation, Uppsala University (2012). URL http://uu.diva-portal.org/smash/ record.jsf? searchId=1\&pid= diva2:564033.

[3] J. Kjellin, Vertical axis wind turbines: Electrical system and experimental results, dissertation, Uppsala University (2012). URL http://uu.diva-portal.org/smash/ record.jsf?pid=diva2:559793.

[4] S. Weekes and A. Tomlin, Evaluation of a semi-empirical model for predicting the wind energy resource relevant to small-scale wind turbines, Renewable Energy 50(2013) 280-288. doi:10.1016/j.renene.2012.06.053.

[5] H. Bergström and S. Söderberg, Wind mapping of sweden - summary of results and methods used, Tech. rep. (2009).

[6] L. Enger, Simulation of dispersion in a moderately complex terrain. part a. the fluid dynamic model, Atmospheric Environment 24A(1990) 2431-2446.

[7] A. Andrén, Evaluation of a turbulence closure scheme suitable for air pollution applications, Journal of Applied Meteorology 29(1990) 224-239.

[8] H. Bergström, A climatological study of boundary layer wind speed using a meso-gammascale higher-order closure model, Journal of Applied Meteorology 35(8) (1996) 1291-1306.

[9] H. Bergström, Boundary-layer modelling for wind climate estimates, Wind engineering 25(5) (2001) 289-299.

[10] W. M. Organization, Guide to Meteorological Instruments and Methods of Observation, 2008 edition, Updated in 2010, Tech. Rep. WMO-No. 8, Geneva, Switzerland (2012).

[11] S. Emeis, Wind Energy Meteorology, Springer, 2013.

[12] M. Raupach, Simplified expressions for vegetation roughness length and zero-plane displacement as functions of canopy height and area index, Boundary-Layer Meteorology (1994) 211-216.

[13] M. L. Thøgersen, M. Motta, T. Sørensen and P. Nielsen, Measure-correlate-predict methods: Case studies and software implementation, in: EWEA Conference Proceedings, EMD International A/S, 2007.

[14] R. Lucchesi, File specification for MERRA products. GMAO office note no. 1 (version 2.3), Tech. rep. (2012). URL Http://gmao.gsfc.nasa.gov/pubs/officenotes.

[15] N. Earl, S. Dorling, R. Hewston and R. von Glasow, 1980-2010 variability in U.K. surface wind climate, Journal of Climate 26 (4) (2013) 1172-1191. doi:10. 1175/JCLI-D-1200026.1 . 
